\title{
Qingre Huoxue Decoction, A Traditional Chinese Herbal Formulation, Impacts Angiogenesis in Psoriasis-Like Skin Through the HIF-1a/Flt-1/VEGF Pathway
}

\author{
Wen Li \\ Shuguang Hospital \\ Xiang He \\ Shuguang Hospital \\ Jiong Zhu \\ Shuguang Hospital \\ Huimin Zhang (D 13917367207@163.com ) \\ Shuguang Hospital
}

\section{Research Article}

Keywords: Qingre Huoxue Decoction, HIF-1a/FIt-1/VEGF pathway, angiogenesis, psoriasis

Posted Date: December 17th, 2021

DOI: https://doi.org/10.21203/rs.3.rs-1072759/v1

License: () (1) This work is licensed under a Creative Commons Attribution 4.0 International License.

Read Full License 


\section{Abstract}

Background: Qingre Huoxue Decoction (QHD), a traditional Chinese medicine (TCM) formulation, could alleviate psoriasis in our previous studies. The present work aimed to assess QHD's effects on psoriasis and the underpinning mechanism in cultured cells and experimental animals.

Methods: The CCK-8 assay was carried out for cell viability assessment. HUVEC migration was assessed by transwell and wound healing assays. QHD-induced suppression of capillary tube formation in HUVECs was detected by tube formation assay. In addition, the imiquimod (IMQ)-induced male BALB/c mouse model of psoriasis was established to examine the Psoriasis Area and Severity Index (PASI) after QHD administration. HIF-1a, Flt-1 and VEGF expression levels in vivo were assessed by immunoblot, qPCR and immunofluorescence.

Results: The results showed that QHD dose-dependently reduced viability in HUVECs. In addition, QHD suppressed tube formation in HUVECs at levels below those needed to inhibit HUVECs. Upon QHD administration, HUVEC migration was markedly decreased; QHD effectively prevented the migratory ability of HUVECs, as determined by wound areas at $0 \mathrm{~h}, 12 \mathrm{~h}$ and $24 \mathrm{~h}$, respectively. Finally, QHD starkly downregulated HIF-1a, Flt-1 and VEGF in the IMQ-induced mouse model, at the protein and mRNA levels.

Conclusions: In summary, QHD inhibits angiogenesis in cultured cells and mice. HIF-1a/Flt-1/VEGF signaling is important in angiogenesis and psoriasis development. These findings provide a rationale for developing QHD for clinical use against psoriasis.

\section{Introduction}

Psoriasis represents an incurable chronic inflammatory skin disease affecting about $3 \%$ of all humans ${ }^{1}$. In Caucasians, an incidence of about 100,000 people per year has been reported. Histologically, psoriasis mostly features enhanced angiogenesis, unrestrained growth, abnormal keratinocyte differentiation and high amounts of infiltrated immune cells ${ }^{2,3}$. However, how psoriasis is initiated remains unknown. It was suggested that angiogenesis represents a hallmark of psoriasis and psoriatic arthritis ${ }^{4}$. Meanwhile, angiogenesis is accompanied by enhanced endothelial cell (EC) proliferation, adhesion and migration ${ }^{5}$.

Critical angiogenic factors, including vascular endothelial growth factor (VEGF) and its receptor Flt-1, show increased amounts in human psoriasis skin ${ }^{6}$. Mounting evidence indicates vascular endothelial growth factor (VEGF) upregulation constitutes an early and essential factor in psoriasis pathogenesis. It was recently demonstrated hypoxia-induced VEGF is a hallmark of psoriasis. In agreement, after psoriasis treatment with a TNF inhibitor, VEGF and Flt-1 are downregulated in P-DMSCs, and angiogenesis is reduced in the skin lesion. Hypoxia is characteristic of the skin tissue in patients with psoriasis, with HIF in keratinocytes and endothelial cells promoting angiogenesis by increasing the expression of VEGF and Flt-1, respectively. In psoriasis, abnormal rapid cell proliferation increases oxygen 
consumption, while epidermal thickening reduces oxygen supply. It was suggested local hypoxia in skin might be critical for adaptive upregulation of angiogenic proteins, including hypoxia inducible factor-1 alpha (HIF-1a).

The traditional Chinese herbal formulation Qingre Huoxue Decoction (QHD) could alleviate psoriasis in our previous work. QHD is mainly composed of Sophora japonica (Huaihua), Angelica sinensis (Danggui), Sparganium stoloni erum (Sanleng), Curcuma zedoaria (Ezhu) and Spatholobus suberectus Dunn (Jixu eteng). The main pharmacologically relevant constituents include Rytub (Huaihua), ferulic acid (Danggui), isocurcumenol (Ezhu), hexadecanoic acid (Sanleng) and formononetin (Jixueteng). To determine whether QHD modulates angiogenesis by regulating hypoxia during psoriasis treatment, the imiquimod-induced psoriasis-like mouse model was used. Angiogenesis and related genes were detected in mouse psoriasis-like lesions induced by imiquimod. The present work aimed to assess QHD's effects on psoriasis and the underpinning mechanism in cultured cells and experimental animals.

\section{Methods}

All assays involving animals had approval from the Ethics Committee of Shanghai University of Traditional Chinese Medicine.

\section{Drug preparation}

Drug components were provided by Jiangyin Tianjiang Pharmaceutical (China). After boiling the herbs, the filtrates underwent concentration and drying under vacuum at $60^{\circ} \mathrm{C}$. The resulting extract was further lyophilized to produce QHD (yield of $27.5 \%)^{7}$. High performance liquid chromatography (HPLC) was utilized for measuring the main constituents. The drug composition ratios of Sophora japonica (Huaihua), Angelica sinensis (Danggui), Sparganium stoloni erum (Sanleng), Curcuma zedoaria (Ezhu) and Spatholobus suberectus Dunn (Jixu eteng) in QHD were 40,20,20, 10 and 10\%, respectively. QHD was solubilized with PBS. The final extract (stored at $4^{\circ} \mathrm{C}$ ), was submitted to quality control per the guidelines published by Chinese State Food and Drug Administration (SFDA).

Cells and culture

Human Umbilical Vein Endothelial Cell (HUVEC; Lonza, Belgium) culture was carried out with endothelial basal medium (EBM) containing the components of Endothelial cell growth medium-2 Bullet Kit (Lonza) according to instructions. All cells were cultured at $37^{\circ} \mathrm{C}$ in a humid environment containing $5 \% \mathrm{CO} 2$.

\section{Animal experiments}

Assays involving animals had approval from the ethics committee of Shuguang Hospital affiliated to Shanghai University of Traditional Chinese Medicine. Totally 25 SPF BALB/c male mice, $18 \pm 2 \mathrm{~g}$, were provided by Shanghai Slac Laboratory Animal (China; license SCXK [Shanghai] 2008-0016) and housed in Shanghai University of Traditional Chinese Medicine (laboratory license, SCHK [Shanghai] 2021-0016). 
The twenty-five animals were randomized into five groups, including the IMQ (IMQ, psoriasis model), IMQ+QHD low-dose (IMQ+L, 8 mg/kg/day), IMQ+QHD medium-dose (IMQ+M, 16 mg/kg/day), IMQ+QHD high-dose (IMQ+H, $32 \mathrm{mg} / \mathrm{kg} /$ day) and methotrexate (MTX, $1 \mathrm{mg} / \mathrm{kg} /$ day) groups. Totally $42 \mathrm{mg}$ of IMQ cream was administered daily to the animals (back skin) for 7 days. Erythema and scaling were scored at 0, 2, 4 and 7 days, respectively, as 0 (none), 1 or2 (moderate), 3 (severe) and 4 (very severe) according to the Psoriasis Area Severity Index (PASI). On the eighth day, euthanasia was performed by intravenous injection of excess pentobarbital $100 \mathrm{mg} / \mathrm{kg}$, followed by skin sample collection for hematoxylin and eosin (H\&E) staining and Western blot. Figure.3 shows the experimental design.

The CCK-8 assay

The CCK-8 assay was carried out for cell viability assessment. Cell seeding was performed in 96-well plates at $10^{5}$ cells per well followed by a $24-\mathrm{h}$ incubation at $37^{\circ} \mathrm{C}$ prior to the administration of QHD at 0 , $25,50,100,150$ and $200 \mu \mathrm{g} / \mathrm{ml}$ for $48 \mathrm{~h}$. Post-treatment, $0.5 \mathrm{mg} / \mathrm{ml} \mathrm{CCK}-8$ solution ( $100 \mu \mathrm{l} \mathrm{per} \mathrm{well)} \mathrm{was}$ supplemented for a 2-h incubation. OD was read on a Varioskan Flash multiscan plate reader (Thermo, USA) at $450 \mathrm{~nm}$. Triplicate assays were carried out.

Transwell migration assay

HUVECs migration was assessed in 24-well Transwell plates using 8- $\mu \mathrm{m}$-pore polycarbonate membrane inserts (Corning, USA) with Matrigel (BD Biosciences) coating as directed by the manufacturer. Briefly, $3 \times 10^{5}$ cells in $100 \mu l$ medium were placed in each upper compartment for a 24 -h incubation at $37^{\circ} \mathrm{C}$. Cells detected on the lower surface of the membrane by hematoxylin staining were numbered in five randomly selected high-power fields. Triplicate assays were carried out.

Scratch assay

The scratch assay was performed to further examine HUVEC migration as reported previously ${ }^{8}$. In brief, cells were treated for $48 \mathrm{~h}$ and a sterile $200-\mu \mathrm{l}$ micropipette tip was used for scratching the well's center. Upon washing with PBS, EBM with $1 \%$ FBS (Gibco Life Technologies, USA) was supplemented. The samples were incubated at $37^{\circ} \mathrm{C}$, and gaps were examined at 0,12 and $24 \mathrm{~h}$, respectively, using an inverted microscope equipped with an Olympus DP70 camera (Olympus, Japan). Image $\mathrm{J}$ was utilized for quantitation.

Tube formation assay

HUVECs were examined for tube formation with Geltrex-reduced growth factor basement membrane matrix (Invitrogen), as directed by the manufacturer. For quantifying tube formation, an inverted microscope (Olympus) was utilized to count meshes which were further analyzed with Image $\mathrm{J}$.

Real-time quantitative PCR 
Total RNA extraction utilized TRIzol reagent (Invitrogen) as directed by the manufacturer. A reverse transcription kit from Takara was used for cDNA synthesis, followed by quantitative PCR with SYBR green (Takara). The following primers were utilized: HIF-1a, sense 5'-CATCTCCATCTCCTACCCACAT-3' and antisense 5'-ACTCCTTTTCCTGCTCTGTTTG-3'; VEGF, sense 5'-TGGGCTCTTCTCGCTCCGTAGTAG-3' and antisense 5'-GCCGCCTCACCCGTCCAT-3'; Flt-1, sense 5'-AAAGGCTGAGCATCACTCCC-3' and antisense 5'CTACAGGTGTAGAGGCCCGT-3'. The $2^{-\Delta \Delta} \mathrm{Ct}$ method was used for the analysis of triplicate assays.

Immunoblot

Prior to protein extraction, imiquimod (IMQ)-induced mice were treated with QHD for 24h. Total protein was extracted from IMQ-induced mouse samples using a protein extraction kit (Applygen Technologies, China) as directed by the manufacturer. Protein separation was carried out by $12 \%$ SDS-PAGE, followed by transfer onto NC membranes. These membranes underwent overnight incubation $\left(4^{\circ} \mathrm{C}\right)$ with anti-HIF1a (1:1000, Novus Biologicals, USA), anti- $\beta$-actin (1:5000, Cell Signaling Technology, CST, USA), VEGF (1:5000, CST) and Flt-1 (1:2000, CST), respectively. This was followed by treatment with a 24-h incubation with secondary antibodies at $4^{\circ} \mathrm{C}$. The enhanced chemiluminescence system was utilized for detection. Densitometric analysis was performed with the Quantity One image analysis software (BioRad, USA).

\section{Statistical analysis}

GraphPad Prism 6 (GraphPad Software, USA) was used for data analysis. Student's $t$ test and Wilcoxon's signed rank test were performed for comparisons. $P<0.05$ reflected statistical significance. Data are mean \pm standard deviation (SD).

\section{Results}

QHD inhibits proliferation and capillary tube formation in HUVECs

We evaluated QHD's effects on HUVEC proliferation by the CCK-8 assay. It was found QHD dosedependently reduced proliferation in HUVECs (Figure.1A). Following a 24-h treatment, QHD's IC50 in HUVECs averaged $471.5 \mu \mathrm{g} / \mathrm{ml}$. Primary HUVECs incubated for 24 adhered to the plate. Following a 3 to $5-$ day incubation, corroborating previously reported findings, HUVECs showed cobblestone-like morphology. What's more, the angiogenic capability of HUVECs was dose-dependently altered by QHD. QHD at 20, 200 and $400 \mu \mathrm{g} / \mathrm{ml}$ for $24 \mathrm{~h}$, respectively, markedly reduced tube formation in HUVECs in comparison with untreated cells $(P<0.05)$ (Figure.1B). Jointly, the above data demonstrated QHD suppressed tube formation in HUVECs at levels starkly lower than needed for inhibiting HUVECs. Remarkably, QHD promoted capillary tube formation in HUVECs.

QHD suppresses HUVEC migration in vitro 
Endothelial cell migration is important in neovascularization, and involves chemotactic, haptotactic and mechanical components. HUVEC migration was first assessed by the transwell assay (Figure.2A). In comparison with control cells, the QHD groups of HUVECs had starkly reduced migration. Next, HUVECs were examined for mechanical migration by the scratch assay. At 0,12 and $24 \mathrm{~h}$ upon wounding, respectively, shorter distances were travelled by HUVECs after QHD treatment (Figure.2B). These data also demonstrated that QHD effectively reduced the migratory ability of HUVECs.

QHD impacts angiogenesis through the HIF-1a/FIt-1/VEGF pathway in the imiquimod (IMQ)-induced mouse model

In the process of angiogenesis, HIF-1 a/Flt-1/VEGF signaling plays a critical role. To further assess QHD's effects, the IMQ-induced mouse model was established. At 3-5 days, mouse recombinant QHD was given by gavage. On the seventh day, psoriasis-like skin inflammation was observed as reflected by erythema and scaling (Figure.3). In this study, QHD-reduced angiogenesis was investigated using dorsal skin images of BALB/c mouse psoriasis models (Figure.3).

Skin samples from animals administered QHD at 200 and $400 \mu \mathrm{g} / \mathrm{kg}$ were thinner, with reduced neovascularization compared with the model group. Its effect was similar to that of the positive control drug. To detect the cell expression levels and localizations of HIF-1 a and Flt-1 in the IMQ-induced mouse model, immunofluorescence was performed (Figure.4A-B). In addition, HIF-1a, Flt-1 and VEGF protein levels in the IMQ-induced mouse model were assessed by Western blot (Figure.4C). Finally, HIF-1a, Flt-1 and VEGF mRNA amounts in the IMQ-induced mouse model were assessed by qRT-PCR (Figure.4D-F). As shown in Figure.4, QHD remarkably inhibited the protein and mRNA expression levels of these effectors in the IMQ-induced mouse model.

\section{Discussion}

It was found that local hypoxia is an important feature of psoriasis. Hypoxia is a major factor in the early pathogenetic events of psoriasis, contributing to inflammatory cell recruitment; meanwhile, the cross-talk involving epidermal and immune cells likely control psoriasis development. Therefore, adjustment of the hypoxic microenvironment is an important direction in the treatment of psoriasis. Angiogenesis is one of the major mechanisms of tissue adaptation to hypoxia.

It is admitted local hypoxia in skin is essential in the adaptive induction of angiogenesis, upregulating multiple effectors such as hypoxia inducible factor-1 alpha (HIF-1a). Multiple reports have revealed HIF$1 \mathrm{a}$ is upregulated in psoriatic skin lesions and in blood.

HIF- $1 \mathrm{a}$ is a heterodimeric transcriptional complex comprising the HIF-1a and HIF- $1 \beta$ subunits, with a function in controlling oxygen levels ${ }^{9,10}$. However, HIF-1 was recently shown to affect other stress response events beside hypoxia. Meanwhile, HIF-1a amounts are elevated in psoriatic lesions ${ }^{11}, 12$, and in serum specimens from psoriasis cases. These elevated HIF-1 a amounts alongside a high association of HIF-1a upregulation with disease severity suggest a major role for HIF-1a in psoriasis' pathogenetic 
mechanisms ${ }^{13}$. Therefore, angiogenesis, exaggerated proliferation and inflammatory response might constitute a vicious circle, with HIF-1a helping maintain and amplify this vicious circle. In agreement, drugs controlling HIF-1a expression might be efficient in disease management. HIF-1a could represent an important target to control inflammatory response and angiogenesis, since many effectors in various pathways seem to involve HIF-1a.

Traditional Chinese medicinal (TCM) preparations have been effectively utilized in treating psoriasis for years. Recently, angiogenesis in psoriasis was shown to be suppressed by TCM preparations. The present study showed QHD inhibits proliferation, migration and tube formation in cultured HUVECs. The present data also demonstrated QHD can significantly reduce the PASI in IMQ-induced mouse models. Multiple preliminary works showed angiogenesis is associated with the HIF-1a/FIt-1/VEGF pathway. The IMQinduced mouse model was established to further explore the mechanism underpinning the inhibitory effects of QHD on psoriasis. The present in vivo study showed that QHD effectively suppresses angiogenesis, which is associated with the HIF-1 a/FIt-1/VEGF pathway.

\section{Conclusions}

In summary, this study firstly reported associations of HIF-1a, Flt-1 and VEGF with psoriasis via QHD intervention. These findings indicate QHD inhibits angiogenesis both in cultured cells and in mice. HIF1a/FIt-1/VEGF signaling is critical for angiogenesis, even in psoriasis. The TCM preparation QHD could inspire a rational development of new therapeutic options for clinical psoriasis. However, the molecular mechanism behind QHD's effect on the HIF-1a/FIt-1/VEGF pathway is unclear, which deserves further investigation.

\section{Declarations}

\section{Ethics approval}

All animal studies were approved by the ethics committee of the Shuguang Hospital affiliated to Shanghai University of Traditional Chinese Medicine and the principles of laboratory animal care were followed in all animal experiments. The study was carried out in compliance with the ARRIVE guidelines.

Consent for publication

The authors declare that they consent for publication.

Availability of data and materials

All data generated or analysed during this study are included in this published article.

Competing interests

The authors declare that they have no competing interests. 
Funding

This work was supported by the projects of the National Natural Science Foundation of China 81974570) and Shuguang Hospital Affiliated to Shanghai University of TCM(SGKJ 201804).

Authors' contributions

Conception and design: Wen Li, Xiang He

Financial support: Huimin Zhang, Jiong Zhu

Provision of study materials or patients: Wen Li, Jiong Zhu

Collection and assembly of data: Wen Li, Jiong Zhu

Data analysis and interpretation: Wen Li, Xiang He Huimin Zhang

Manuscript writing: All authors

Acknowledgements

We would like to thank all the experts who were willing to take part in the time-consuming survey rounds and who contributed to a lively exchange with their many comments.

\section{References}

1. Crow JM. Psoriasis uncovered. Nature 2012;492:550-1.

2. Lowes M, Bowcock A, Krueger J. Pathogenesis and therapy of psoriasis. Nature 2007;445:866-73.

3. Papp KA, Blauvelt A, Bukhalo M, Gooderham M, Padula SJ. Risankizumab versus Ustekinumab for Moderate-to-Severe Plaque Psoriasis. New England Journal of Medicine 2017;376:1551-60.

4. Detmar M, Brown LF, Claffey KP, Yeo KT, Dvorak HF. Overexpression of vascular permeability factor/vascular endothelial growth factor and its receptors in psoriasis. Journal of Experimental Medicine 1994;180:1141-6.

5. Shweiki D, Itin A, Soffer D, Keshet E. Vascular endothelial growth factor induced by hypoxia may mediate hypoxia-initiated angiogenesis. Nature 1992;359:843-5.

6. Wang W, Yuhai, Wang H, Chasuna, Bagenna. Astilbin reduces ROS accumulation and VEGF expression through Nrf2 in psoriasis-like skin disease. Biol Res 2019;52:49.

7. Hou F, Wen L, Qi S, Li H, Jian X. Yi Ai Fang, a traditional Chinese herbal formula, impacts the vasculogenic mimicry formation of human colorectal cancer through HIF-1 $a$ and epithelial mesenchymal transition. BMC complementary and alternative medicine 2016;16.

8. Niu X, Han Q, Liu Y, Li J, Zhang K. Psoriasis-associated angiogenesis is mediated by EDIL3. Microvascular Research 2020;132:104056. 
9. Hsu TS, Lin YL, Wang YA, Mo ST, Chi PY, Lai AC et al. HIF-2a is indispensable for regulatory T cell function. Nat Commun 2020;11:5005.

10. Wang GL, Jiang BH, Rue EA, Semenza GL. Hypoxia-inducible factor 1 is a basic-helix-loop-helix-PAS heterodimer regulated by cellular 02 tension. Proc Natl Acad Sci U S A 1995;92:5510-4.

11. Kim JH, Bae HC, Kim J, Lee H, Ryu W-I, Son ED et al. HIF-1a-mediated BMP6 down-regulation leads to hyperproliferation and abnormal differentiation of keratinocytes in vitro. Experimental Dermatology 2018;27:1287-93.

12. Rosenberger $C$, Solovan $C$, Rosenberger $A D$, Jinping L, Treudler R, Frei $U$ et al. Upregulation of hypoxia-inducible factors in normal and psoriatic skin. J Invest Dermatol 2007;127:2445-52.

13. Zhu WJ, Li P, Wang L, Xu YC. Hypoxia-inducible factor-1: A potential pharmacological target to manage psoriasis. International Immunopharmacology 2020;86:106689.

\section{Figures}

A

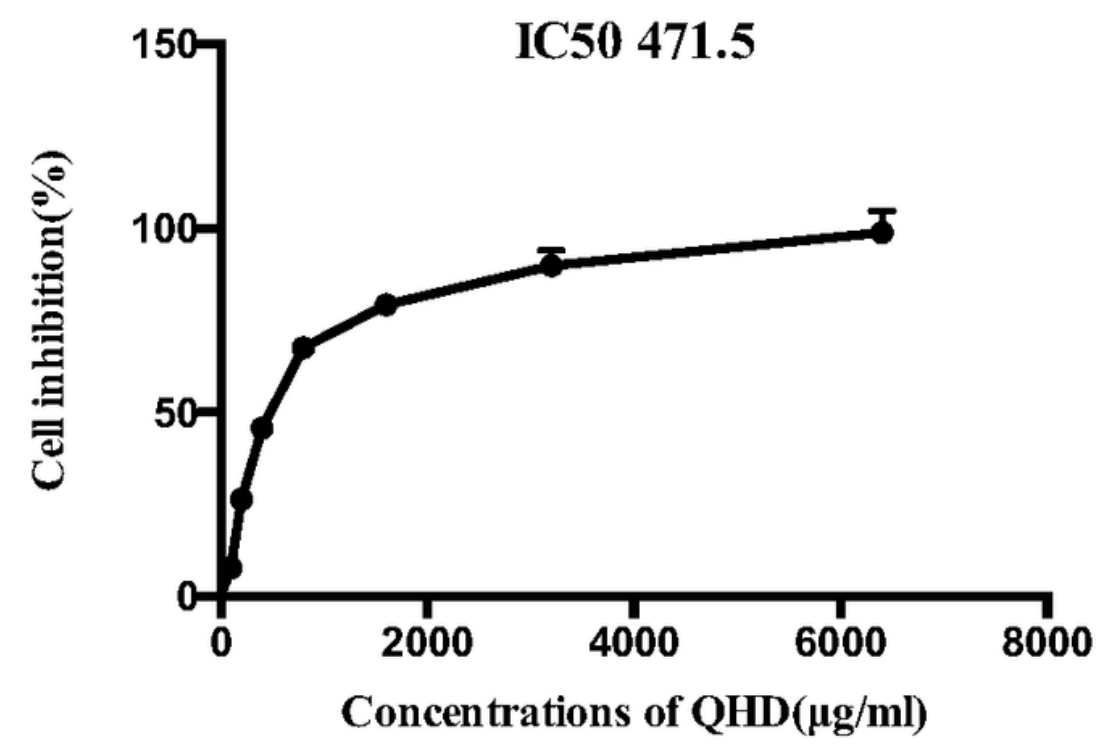

B

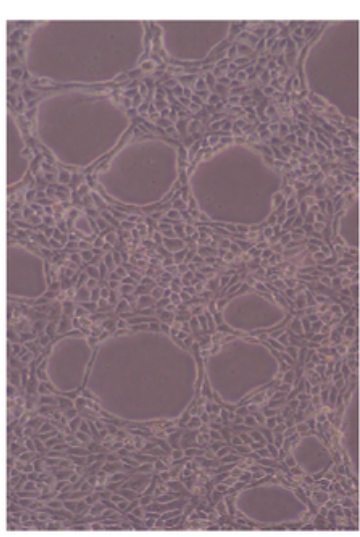

ctrl

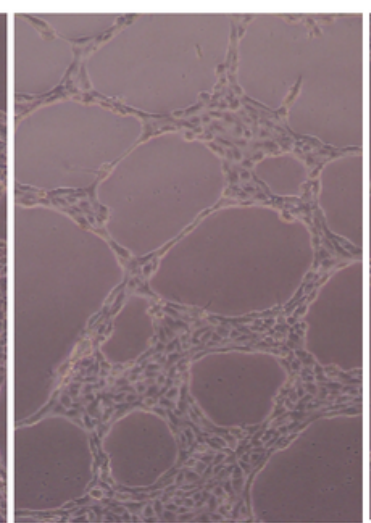

QHD-L

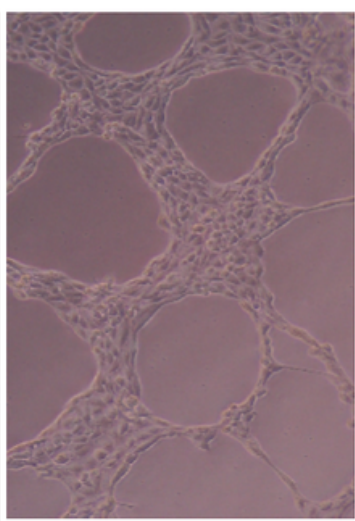

QHD-M

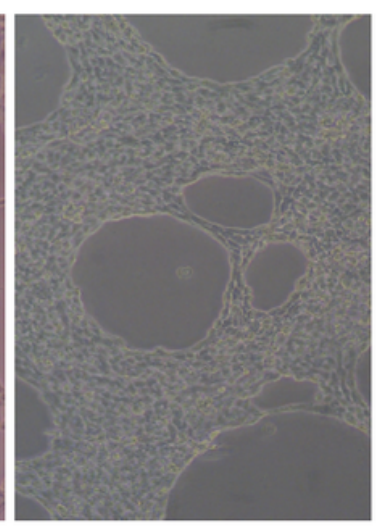

QHD-H

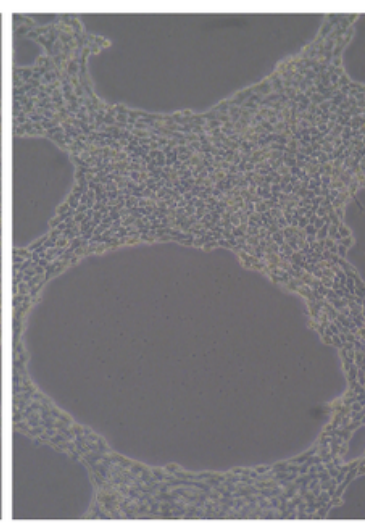

MTX 


\section{Figure 1}

QHD inhibited proliferation of HUVECs cells and depressed the information of capillary tube in HUVECs cells. A: QHD reduces the viability of HUVECs cells. The cells were incubated with QHD at $0,100,200,400$, $800,1600,3200$ and $6400 \mu \mathrm{g} / \mathrm{ml}$ for $48 \mathrm{~h}$. The viable cells were determined using CCK-8 assay. Data presented were means \pm SD of three independent experiments. ${ }^{*} P<0.05$ compared with the control group. B: The angiogenesis capability of HUVECs cell in vitro is affected by QHD. HUVECs cells were treated with QHD at 20,200 and $400 \mu \mathrm{g} / \mathrm{ml}$ for $28 \mathrm{~h}$, the constitution of QHD was significantly decreased by QHD compared with that of untreated cells and MTX treated cells $\left({ }^{\star} P<0.05\right)$

A

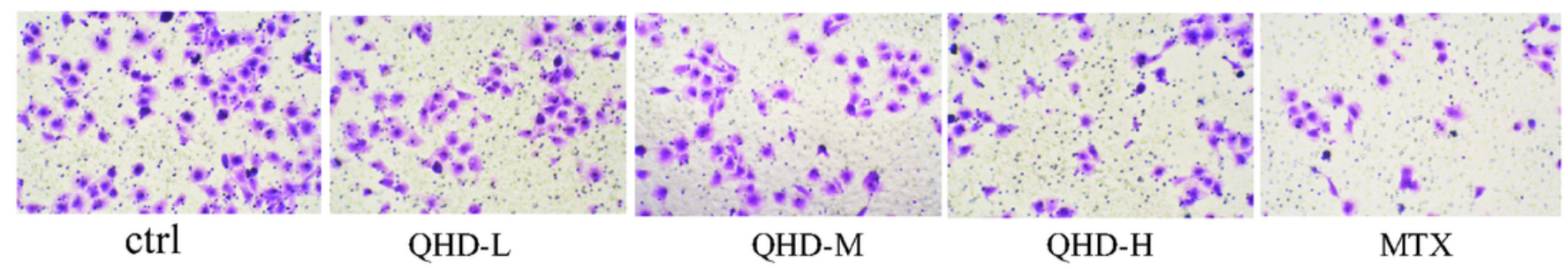

B

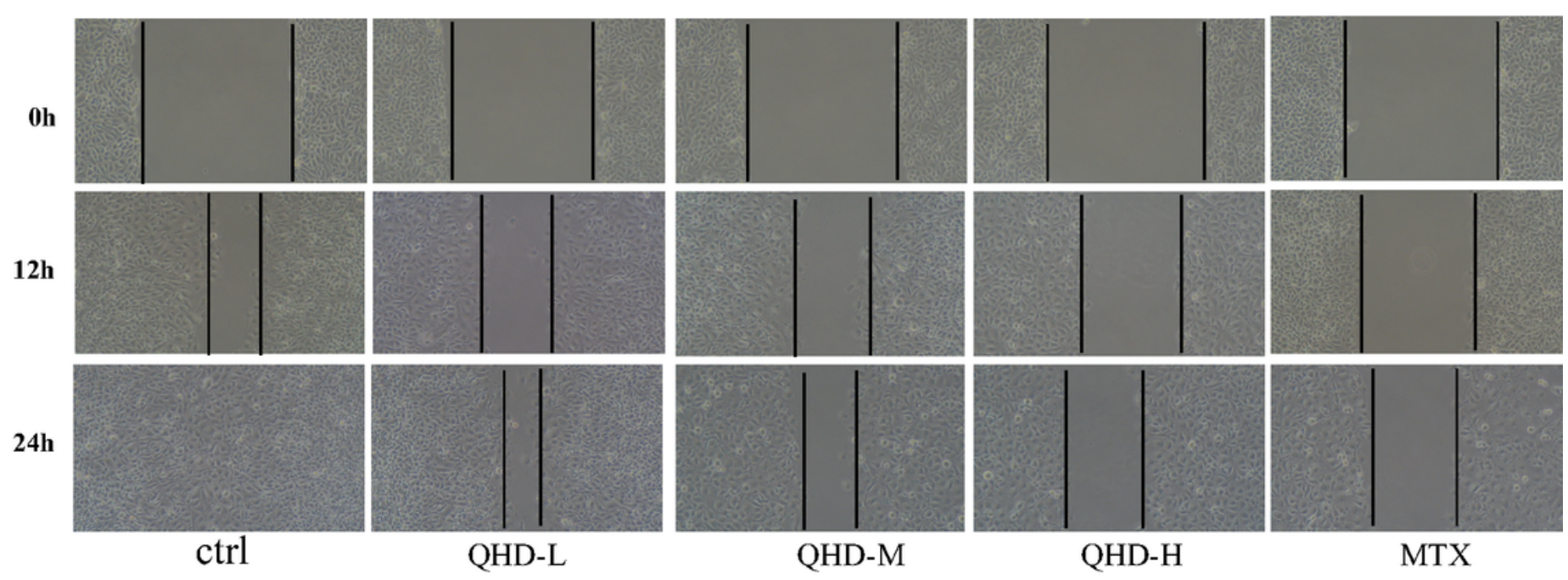

\section{Figure 2}

QHD suppressed migration of HUVECs in vitro. A: The migrated cells towards the lower membranes. The migrated cells towards the lower membranes were stained with $0.1 \%$ crystal violet. Representative photomicrographs of migrated cells in control, QHD-L, QHD-M, QHD-H and MTX groups. (magnification 10x). QHD can significantly suppressed the migration of HUVECs. $P<.005 \mathrm{vs.} \mathrm{control} \mathrm{and} \mathrm{MTX} \mathrm{groups.}$ $\mathrm{B}$ :The wound scratch assay at 0,12 and $24 \mathrm{~h}$ was recorded. The distance of migrated cells travelled in each treatment group was shown. The area of gap was of gap quantified at $0 \mathrm{~h}$ and $12 \mathrm{~h}$ in different groups. Calculated formula: the area of gap $(\%)=1$ - area of $12 \mathrm{~h} /$ area of $0 \mathrm{~h}$. QHD can significantly inhibit the migration capacity of HUVECs. $P<.005$ vs. control and MTX groups. 


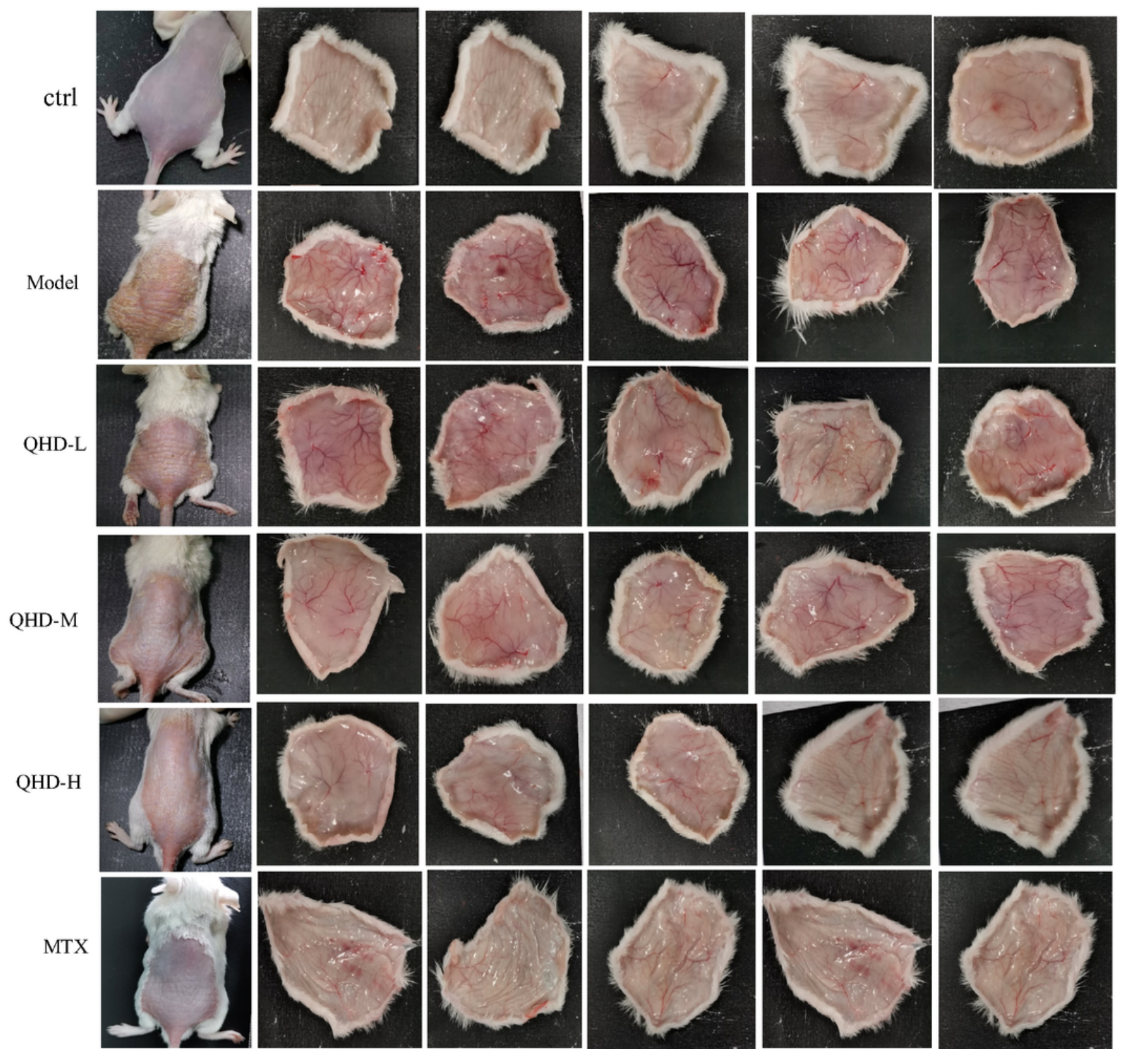

Figure 3

The Dorsal Skin Images of BALB/c Mouse Psoriasis Models Induced by Imiquimod. Psoriasis-like skin inflammation was induced to the back skin of the mice characterized by erythema and scaling.

QHD reduced angiogenesis was investigated through the dorsal skin images of BALB/c mouse psoriasis models induced by imiquimod. Statistical difference was analyzed by Student's t-test. ${ }^{*} P<0.05$ compared with that of control group and MTX group. 
A
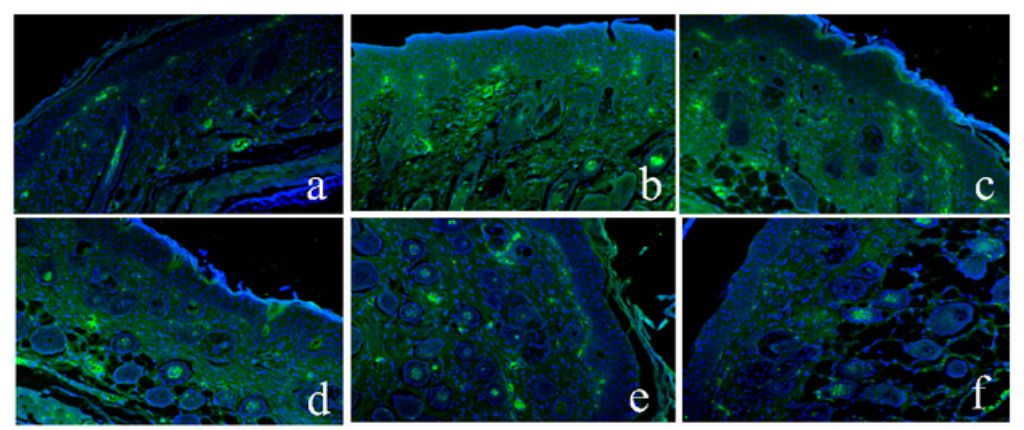

$\mathrm{B}$
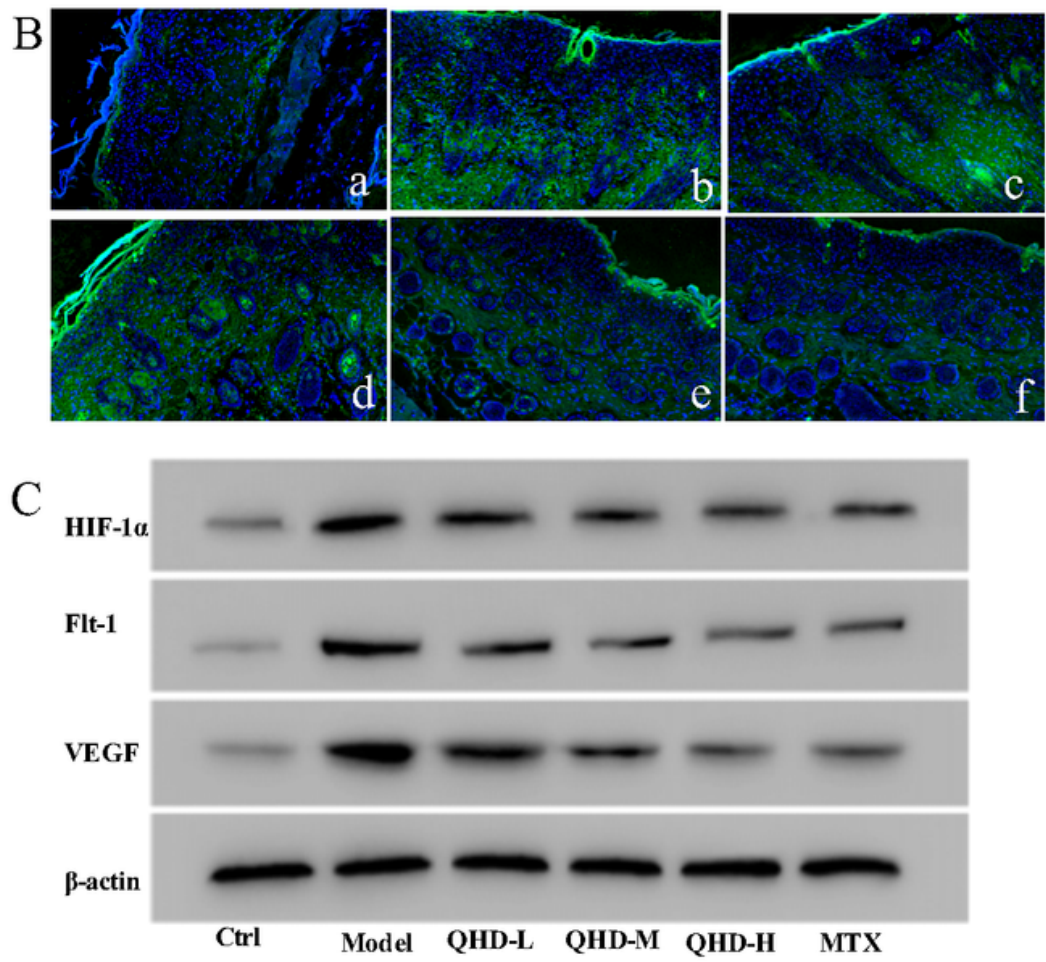

$\mathrm{D}$

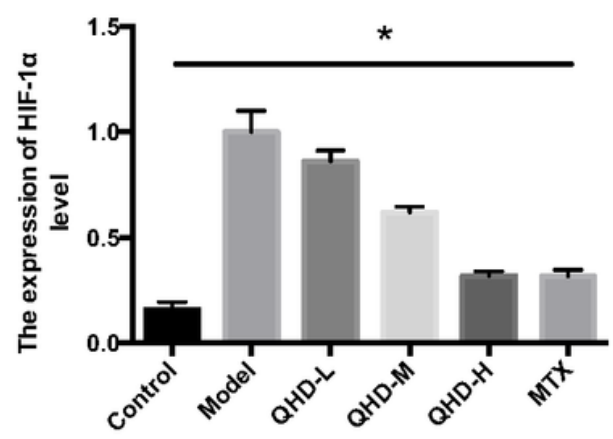

$\mathrm{E}$

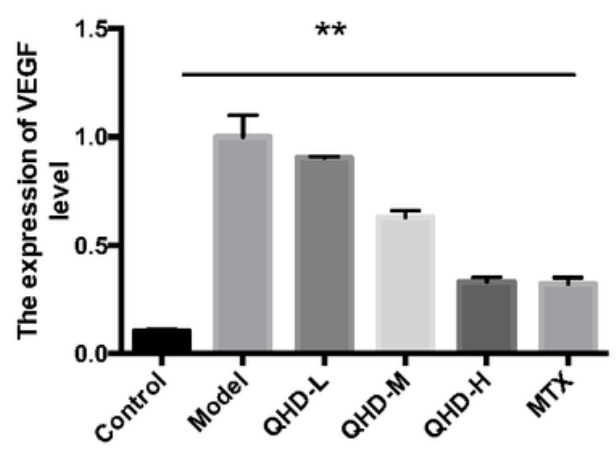

F

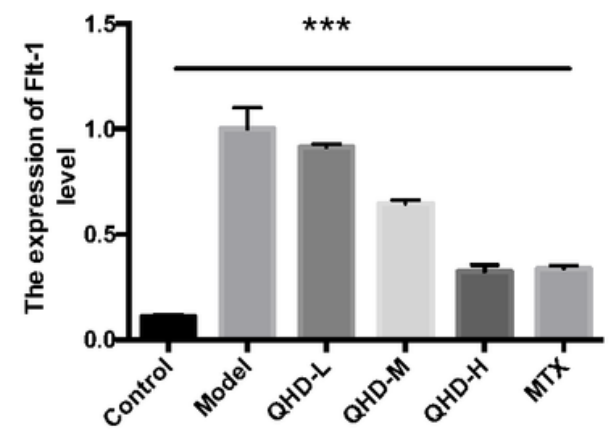

\section{Figure 4}

QHD impacts angiogenesis through HIF-1a/FIt-1/VEGF signal pathway in imiquimod (IMQ)-induced mouse model. A-B: The expression levels and expression site in cells of HIF-1a and Flt-1 in IMQ-induced mouse model by immunofluorescence. C: The protein expression of HIF-1a, Flt-1 and VEGF in IMQinduced mouse model and negative control groups. D-F: HIF-1a, Flt-1 and VEGF mRNA levels were normalized to $\beta$-actin. The HIF-1a, Flt-1 and VEGF relative genes expression levels were calculated using the $2-\Delta \Delta$ CT method. $P<.05$ was considered to be statistically significant. Graph shows mean $\pm \mathrm{SD}$. * $p<$ $.05, * \star p<.01, * \star * p<.05$. 